\title{
„... darum rufe ich jetzt den Rettungsdienst!“
}

\section{Eine qualitative Studie zu Notfallszenarien in Pflegeheimen}

\author{
Juliane Poeck ${ }^{1}$ (D), Carsten Bretschneider ${ }^{1}$, Silke Freihoff2,3, Andreas Günther ${ }^{4}$, \\ Martina Hasseler ${ }^{5}$, Nils Schneider², Jutta Bleidorn', Sven Schwabe² \\ ${ }^{1}$ Institut für Allgemeinmedizin, Universitätsklinikum Jena \\ ${ }^{2}$ Institut für Allgemeinmedizin, Medizinische Hochschule Hannover \\ ${ }^{3}$ Klinik für Rehabilitationsmedizin, Medizinische Hochschule Hannover \\ ${ }^{4}$ Fachbereich Feuerwehr, Stadt Braunschweig \\ ${ }^{5}$ Fakultät Gesundheitswesen, Ostfalia Hochschule für angewandte Wissenschaften - Hochschule Braunschweig / Wolfenbüttel
}

\begin{abstract}
Zusammenfassung: Hintergrund: Notfälle in Pflegeheimen führen zu einer hohen Anzahl von Rettungsdiensteinsätzen und Krankenhauszuweisungen. Eine umfassende interprofessionelle Perspektive auf Notfälle in Pflegeheimen ist bislang wenig untersucht. Ziel: Charakterisierung, Identifizierung und Priorisierung von Notfallszenarien nach Relevanz und Häufigkeit in Pflegeheimen. Methoden: Zwei multimethodale berufsgruppenübergreifende Gruppendiskussionen wurden mit insgesamt 18 Teilnehmenden aus Pflege, Medizin und Wissenschaft im Januar und Februar 2020 durchgeführt. Die Gruppendiskussionen wurden aufgezeichnet, transkribiert und qualitativ-inhaltsanalytisch nach Mayring ausgewertet. Ergebnisse: Notfallszenarien in Pflegeheimen entstehen aus einem Zusammenspiel von personenbezogenen Anlässen und Kontextbedingungen. Als wesentliche personenbezogene Anlässe wurden genannt: Sturz, entgleiste Vitalwerte, auffälliges Verhalten, neurologische Symptome und Leblosigkeit. Kontextbedingungen gliedern sich in organisatorisch-strukturelle, politisch-rechtliche und ethische Aspekte. Als besonders relevant stellten sich unzureichende Kommunikation zwischen den Akteuren, Unsicherheiten beim Personal, fehlende Patientenverfügungen und Arbeitsverdichtung in der Pflege heraus. Schlussfolgerungen: Notfälle in Pflegeheimen stellen sich als komplexe Notfallszenarien dar. Kontextbedingungen sind bedeutsam für den Umgang mit Notfallszenarien in Pflegeheimen. Handlungsempfehlungen sollten sich an der Perspektive der in der Notfallversorgung beteiligten Akteure orientieren und die Kontextbedingungen stärker berücksichtigen.
\end{abstract}

Schlüsselwörter: Pflegeheime, Notfälle, Geriatrie, interprofessionelle Zusammenarbeit, qualitative Methoden

\section{"... that's why I call the ambulance!" - A qualitative study of emergency scenarios in nursing homes}

Abstract: Background: In nursing homes, emergencies lead to frequent utilisation of emergency medical services (EMS) and emergency department visits. A broad interprofessional perspective of involved practitioners on emergencies in nursing homes has been little studied so far. Aim: Characterization, identification and prioritization of emergency scenarios by relevance and frequency in nursing homes. Methods: We conducted two multi-method, interprofessional group discussions with a total of 18 participants from nursing, medicine and science in January and February 2020. Group discussions were recorded, transcribed and analyzed using qualitative content analysis according to Mayring. Results: Emergency scenarios in nursing homes arise from interactions between person-related aspects and contextual conditions. The following person-related aspects were named as relevant: Falls, unstable vital signs, abnormal behavior, neurological symptoms and lifelessness. Contextual conditions are classified into organizational-structural, political-legal and ethical aspects. The following were considered to be most relevant: lack of communication between the actors, uncertainties among staff, absent living wills and increasing workload in nursing. Conclusions: Emergencies in nursing homes turn out to be complex emergency scenarios. Contextual conditions are important for dealing with emergency scenarios in nursing homes. Recommended actions should be based on the perspective of involved practitioners on emergencies and take greater account of the contextual conditions.

Keywords: Nursing homes, emergencies, geriatrics, interprofessional relations, qualitative methods 
Was ist (zu dieser Thematik) schon bekannt?

Notfallsituationen in Pflegeheimen führen oft zu vermeidbaren und ungewünschten Krankenhauszuweisungen und stellen in der Notfallversorgung beteiligte Akteure vor Herausforderungen.

\section{Was ist neu?}

Notfallszenarien können mithilfe eines Modells als Zusammenwirken von personenbezogenen Anlässen und Kontextbedingungen konzipiert werden.

Welche Konsequenzen haben die Ergebnisse für die Pflegepraxis?

Handlungsempfehlungen sollten sich an der Perspektive der in der Notfallversorgung beteiligten Akteure orientieren und die Kontextbedingungen stärker berücksichtigen.

\section{Einleitung}

In Deutschland werden über 800000 pflegebedürftige Personen in ca. 14500 Pflegeheimen versorgt (Statista, 2020). Aufgrund der demografischen Entwicklung in der Gesellschaft werden altersassoziierte chronische Erkrankungen immer häufiger und stellen die Versorgungsinstitutionen vor Herausforderungen (Robert Koch-Institut, 2015).

Auftretende Notfälle in Pflegeheimen führen häufig zu Krankenhauszuweisungen der betroffenen Bewohner innen (Kada, Janig, Likar \& Pinter, 2013; Tiedtke et al., 2014). Dabei werden Bewohner_innen fast doppelt so häufig hospitalisiert wie nicht-institutionalisierte Gleichaltrige (Graverholt et al., 2011; Seeger, Ramos \& Hoffmann, 2017; Wang, Shah, Allmann \& Kilgore, 2011). Die kontinuierliche pflegerische Versorgung wird unterbrochen und die Wahrscheinlichkeit negativer Begleiterscheinungen wie Distress, Desorientierung, Verschlechterung existierender Gesundheitsprobleme und Morbidität erhöht (Fernandez, Callahan, Likourezos \& Leipzig, 2008; Morphet, Innes, Griffiths, Crawford \& Williams, 2015; Ouslander, 2019). Gleichzeitig werden zahlreiche Rettungsdiensteinsätze und Krankenhaustransporte aus Pflegeheimen aus medizinischen Gründen als vermeidbar eingeschätzt und entsprechen häufig nicht den Behandlungswünschen der Bewohner_innen (Burke, Rooks, Levy, Schwartz \& Ginde, 2015; Dwyer, Gabbe, Stoelwinder \& Lowthian, 2014; Ouslander et al., 2010).

Als medizinischer Notfall werden laut Behringer, Buergi, Christ, Dodt \& Hogan (2013) ,alle Personen definiert, die körperliche oder psychische Veränderungen im Gesundheitszustand aufweisen, für welche der Patient selbst oder eine Drittperson unverzügliche medizinische und pflegerische Betreuung als notwendig erachtet" (S.625). Die Ersteinschätzung bei Notfällen im Pflegeheim findet durch Pflegefachpersonen statt, die dann entscheiden, in welcher Form sie weitere Hilfe (z. B. Hausärzt_in, Ärztlicher Bereitschaftsdienst, Rettungsdienst) zu Rate ziehen. Die Begriffe „Notfall" und „Notfallsituation" werden gleichgesetzt und primär assoziert mit dem Einsatz des Rettungsdienstes und dem Krankenhaustransport der Bewohner_innen. Die Daten werden häufig retrospektiv mithilfe von Akten- und Krankenkassendatenanalysen erfasst (Burke et al, 2015; Ouslander et al., 2010; Seeger et al, 2017).

Die Forschungsliteratur gibt einen guten Einblick über verschiedene Ursachen für Krankenhauszuweisungen aus Pflegeheimen. Systematische Übersichtsarbeiten identifizierten vor allem traumatische Ereignisse (z.B. Stürze), Veränderungen des mentalen Status, Infektionen, Entzündungen und Atemwegsbeschwerden als häufigste Ursachen für Krankenhauszuweisungen (Lemoyne et al., 2019). Darüber hinaus gibt es eine Diskussion über die Vermeidbarkeit von Rettungsdiensteinsätzen und Krankenhauszuweisungen, in der strukturelle Umstände in den Pflegeheimen als Ursachen für Notfallsituationen benannt werden, z.B. Personalmangel, mangelndes Wissen und fehlende Umsetzung von Advance Care Planning, fehlende Willensbekundungen der Bewohner_innen sowie die eingeschränkte Erreichbarkeit der Hausärzt_innen (Bleckwenn, Bell, Schnakenberg, Weckbecker \& Klaschik, 2019; Chess, Whitman, Croll \& Stefanacci, 2018; Dyntar et al., 2018).

Insgesamt weisen die Befunde daraufhin, dass bei Notfallsituationen in Pflegeheimen mehrere Ebenen zu berücksichtigen sind. Ein ganzheitlicher und interprofessioneller Blick auf Notfallsituationen fehlt bisher. Um diese Komplexität zu eruieren, bedarf es einer berufsübergreifenden Perspektive von Akteuren, die in der Notfallversorgung in Pflegeheimen beteiligt sind (z. B. Pflegefachpersonen, Ärzt_innen, Rettungsdienst).

\section{Ziele und Fragestellungen}

Die vorliegende Studie hat das Ziel, Notfallsituationen in Pflegeheimen aus der berufsübergreifenden Perspektive beteiligter Akteure in der Notfallversorgung (Pflegefachpersonen, Ärzt_innen, Rettungsdienst) zu charakterisieren, zu identifizieren und nach Häufigkeit und Relevanz zu priorisieren. Die Ergebnisse sollen dazu beitragen, Handlungsempfehlungen für ausgewählte Notfallsituationen im Projekt NOVELLE partizipativ zu entwickeln, die Pflegefachpersonen mehr Handlungssicherheit in Notfallsituationen geben.

\section{Methoden}

\section{Kontext und Ethikvotum}

Die vorliegende Studie ist ein Teil des Innovationsfondsgeförderten Projekts NOVELLE „Sektorenübergreifendes und integriertes Notfall- und Verfügungsmanagement für die letzte Lebensphase in der stationären Langzeitpflege“ (Förderkennzeichen: 01NVF18007). Ein positives Ethikvotum der Ethikkommission der Medizinischen Hochschule Hannover vom 27.01.2020 liegt vor (Nr. 8866 BO_K_2020). 


\section{Studiendesign}

Die Studie nutzt ein qualitatives Forschungsdesign zur Rekonstruktion subjektiver Perspektiven und Deutungsmuster von Pflegefachpersonen, Ärzt_innen und dem Rettungsdienst auf Notfallszenarien in Pflegeheimen. Qualitative Methoden können einen Einblick in die Innenperspektive der Beteiligten eröffnen und dadurch kontrolliertes Fremdverstehen ermöglichen (Flick, Kardorff \& Steinke, 2012). Sie eignen sich besonders gut für derart offene und explorative Fragestellungen. Das berufsübergreifende Verständnis von Notfällen kann somit umfassend eruiert werden. Aufgrund der Komplexität der Fragestellungen (Charakterisierung, Identifikation und Priorisierung von Notfallszenarien) wurde ein multimethodaler Ansatz gewählt, bei dem unterschiedliche Formen von Gruppendiskussionen und Moderationstechniken (z.B. Mehrpunktabfrage) im Hinblick auf die Fragestellung miteinander kombiniert worden sind (Bohnsack, 2000; Przyborski \& Riegler, 2010).

\section{Samplingstrategie}

Die Auswahl der Teilnehmenden erfolgte von Oktober 2019 bis Januar 2020 in Stadt und Region Braunschweig. Es wurde eine interprofessionelle Zusammensetzung der Teilnehmenden nach folgenden vorab definierten Kriterien angestrebt (purposeful sampling): a) an der Notfallversorgung in Pflegeheimen beteiligte Pflegefachpersonen aus unterschiedlichen Pflegeheimen, b) an der Notfallversorgung in Pflegeheimen beteiligte Ärzt_innen aus verschiedenen Fachrichtungen (z. B. Allgemeinmedizin, Notfallmedizin), c) vereinzelt Vertreter_innen aus dem Rettungsdienst, d) vereinzelt Wissenschaftler_innen aus dem Gesamtprojekt NOVELLE aufgrund der Verknüpfung zu anderen Teilprojekten. Der Hauptfokus lag in der Rekrutierung vom Pflegefachpersonen und Ärzt_innen.

Es wurde eine Mindesteilnehmeranzahl von 10 Personen pro Termin angestrebt. Dabei sollte die Hälfte der Teilnehmenden durch Pflegefachpersonen vertreten sein.

Das Forscherteam kontaktierte schriftlich und telefonisch einen bereits bestehenden regionalen Arbeitskreis zur Verbesserung der Versorgungssituation in Pflegeheimen und über alle am Gesamtprojekt NOVELLE teilnehmende Pflegeheime (14 Einrichtungen). Alle Interessierten erhielten eine ausführliche Studieninformation, inkl. Informationen zum Datenschutz und -management und gaben ihr schriftliches informiertes Einverständnis zur Teilnahme.

Die Teilnehmenden erhielten pro Termin eine finanzielle Aufwandsentschädigung in Höhe von $150 €$, die Teilnahme an allen Terminen stellte keine Bedingung dar.

\section{Datenerhebung}

Zwei multimethodale Gruppendiskussionen fanden im Januar und Februar 2020 unter Moderation der Autor_innen
JP und SSch statt. Der zeitliche Umfang betrug 180 Minuten pro Termin. Die Inhalte wurden sukzessiv aufeinander aufgebaut. Für die Bearbeitung der Inhalte wurden verschiedene qualitative Methoden und Moderationstechniken eingesetzt.

In multimethodalen Gruppendiskussionen (berufsgruppenheterogene und -homogene Gruppendiskussionen, Mehrpunktabfrage) wurden explorative und themenspezifische Fragestellungen miteinander kombiniert. Die Moderation erfolgte non-direktiv durch die Erstautorin und den Letztautor um viel Raum für Offenheit in der Diskussion zu ermöglichen. In der ersten Gruppendiskussion sollten die Teilnehmenden in berufshomogenen Kleingruppen (1. Ärzt innen, 2. Pflegefachpersonen und Rettungsdienst) ihre Erfahrungen mit Notfällen im Pflegeheim darlegen und Charakteristika derselben zusammentragen unter der Leitfrage „Wodurch sind Notfallsituationen in Pflegeheimen gekennzeichnet?". Ein Gesprächsleitfaden wurde nicht verwendet, um die Diskussionen bewusst offen zu halten. Nach Zwischenauswertung der Ergebnisse erfolgte in der zweiten Sitzung die themenspezifische Diskussion in berufsheterogenen Kleingruppen. Unter Vorgabe der entsprechenden Kategorien wurden weitere personenbezogene Anlässe sowie ethische, politisch-rechtliche und organisatorisch-strukturelle Kontextbedingungen gesammelt und ergänzt. Im Plenum wurden die Ergebnisse in der Gesamtgruppe jeweils verifiziert und konsentiert.

Die Priorisierung der personenbezogenen Anlässe und Kontextbedingungen erfolgte im Termin 2 durch Mehrpunktabfrage im Plenum. Dabei erhielt jede/r Teilnehmende drei Klebepunkte und den Auftrag, diese zu bestimmten Fragestellungen nach subjektiver Einschätzung bestimmten Kategorien zuzuordnen (z. B. „Welche Kategorien sind Ihrer Meinung die häufigsten Kontextbedingungen?"). Die zu bepunktenden Kategorien sind zuvor offen gesammelt und auf Metaplankarten visualisiert worden.

In den Gruppendiskussionen wurden zentrale Diskussionsaspekte von den Moderator_innen auf Metaplankarten zusammengefasst (Ruddat, 2012), visualisiert und an eine Pinnwand geheftet. So wurde sichergestellt, dass alle Teilnehmenden jederzeit die erarbeiteten Ergebnisse einsehen und nachvollziehen konnten. Nach jedem Arbeitsschritt wurden die Teilnehmenden nach Ergänzungen und Anmerkungen gefragt bis ein Konsens erreicht war. Auf diese Weise wurde die Sättigung der Ergebnisse erreicht. Zudem wurden schriftliche Protokolle in den Kleingruppendiskussionen angefertigt. Die Ergebnisvorstellung und -diskussion wurden durch ein Audiogerät aufgenommen, die Dateien wurden regelgeleitet transkribiert und pseudonymisiert.

\section{Datenauswertung}

Die Datenauswertung erfolgte durch die Autor_innen JP, SSch und SF. Die drei Autor_innen waren zum Zeitpunkt der Studie als wissenschaftliche Mitarbeiter_innen angestellt und haben diverse Projekterfahrungen mit qualitativen For- 
schungsmethoden (Erhebung und Auswertung von Einzelinterviews und Gruppendiskussionen). Die Datenanalyse erfolgte mittels qualitativer Inhaltsanalyse nach Mayring (Kuckartz, 2016; Mayring, 2008), unterstützt durch die Software MAXQDA 2018. Die transkribierten Texte wurden systematisch und regelgeleitet auf ihre wesentlichen Inhalte zusammengefasst. Dabei wurden inhaltlich-strukturierende und zusammenfassende Analysetechniken genutzt. Die Entwicklung der zentralen Haupt- und Subkategorien erfolgte in einem induktiv-deduktiven Prozess auf Basis der empirischen Ergebnisse vor dem Hintergrund der Fragestellung. Der überwiegende Teil der Haupt- und Subkategorien wurde deduktiv anhand vorgegebener Ordnungskriterien aus den Fragestellungen, Präsentationen und Protokollen gebildet. Vereinzelte Subkategorien wurden mithilfe der zusammenfassenden Inhaltsanalyse induktiv aus dem Material heraus gebildet (z. B. interne und externe Bedingungen als Subkategorien der organisatorisch-strukturellen Kontextbedingungen). Bei der Auswertung des Datenmaterials wurden nicht eindeutig zuordenbare Codes zwischen den beteiligten Autor_innen offen kommuniziert, diskutiert und konsentiert. Eine Interraterreliabilität wurde nicht berechnet. Das Kategoriensystem (siehe Tab. 2) wurde im interprofessionellen Forscherteam konsentiert.

\section{Ergebnisse}

\section{Sample}

Von den 32 interessierten Personen nahmen insgesamt 18 an der Studie teil. Gründe für die Nicht-Teilnahme waren z.B. fehlende zeitliche Ressourcen oder Terminüberschneidungen. Die Mindestteilnehmeranzahl wurde bei jedem Treffen erreicht und sogar übertroffen. Der Teilnehmerkreis war überwiegend konstant, da 15 von 18 Personen an beiden Terminen teilnahmen. Die Teilnehmenden setzten sich insgesamt zusammen aus acht Pflegefachpersonen (Pflegedienstleitungen und Pflegenden aus sieben Einrichtungen), sechs Ärzt_innen (Allgemeinmedizin, Notfallmedizin, Palliativmedizin, Geriatrie), drei Wissenschaftler_innen (Pflegewissenschaften, Medizininformatik) und ein_e Notfallsanitäter_in. Eine Übersicht, wie sich die Teilnehmenden pro Termin zusammensetzen, ist in Tabelle 1 dargestellt.

\section{Kategoriensystem}

Die Analyse der Daten ergab eine Konzeption von Notfallszenarien sowie ein daran anknüpfendes Kategoriensystem mit zwei Hauptkategorien und je drei Subkategorien (siehe Tab. 2).

Die Konzeption eines komplexen Notfallszenarios als Zusammenwirken von personenbezogenen Anlässen und Kontextbedingungen stellt das Kernergebnis der Arbeit dar (Abb. 1). Die Ergebnisse im Einzelnen werden nachfol-
Tabelle 1. Beschreibung der Teilnehmenden (TN) pro Termin

\begin{tabular}{|c|c|c|c|}
\hline Datum & TN-Zahl & TN-Berufsgruppenverteilung & $\begin{array}{l}\text { Geschlechter- } \\
\text { verteilung }\end{array}$ \\
\hline \multirow[t]{4}{*}{ Termin 1} & 17 & $\begin{array}{l}\text { Ärzt_innen: } \\
6(35,3 \%)\end{array}$ & $\begin{array}{l}7 / 17 \text { männlich } \\
(41,2 \%)\end{array}$ \\
\hline & & $\begin{array}{l}\text { Pflegefachpersonen: } \\
8(47,1 \%)\end{array}$ & $\begin{array}{l}\text { 10/17 weiblich } \\
(58,8 \%)\end{array}$ \\
\hline & & $\begin{array}{l}\text { Notfallsanitäter_in: } \\
1(5,9 \%)\end{array}$ & \\
\hline & & $\begin{array}{l}\text { Wissenschaftler_innen: } \\
2(11,8 \%)\end{array}$ & \\
\hline \multirow[t]{4}{*}{ Termin 2} & 16 & $\begin{array}{l}\text { Ärzt_innen: } \\
4(25,0 \%)\end{array}$ & $\begin{array}{l}\text { 6/16 männlich } \\
(37,5 \%)\end{array}$ \\
\hline & & $\begin{array}{l}\text { Pflegefachpersonen: } \\
8(50,0 \%)\end{array}$ & $\begin{array}{l}\text { 10/16 weiblich } \\
(62,5 \%)\end{array}$ \\
\hline & & $\begin{array}{l}\text { Notfallsanitäter_in: } \\
1(6,2 \%)\end{array}$ & \\
\hline & & $\begin{array}{l}\text { Wissenschaftler_innen: } 3 \\
(18,8 \%)\end{array}$ & \\
\hline
\end{tabular}

gend anhand des Kategoriensystems berichtet und mit Zitaten aus Gruppendiskussionen illustriert.

\section{Charakterisierung von Notfallszenarien}

Für die Teilnehmenden sind Notfälle in Pflegeheimen durch eine Vielzahl unterschiedlicher Merkmale gekennzeichnet. Diese Merkmale tauchen im Versorgungsalltag häufig im Zusammenspiel auf und können als Notfallszenarien konzeptualisiert werden.

Für Pflegefachpersonen können beispielsweise sich verschlimmernde Schmerzen bei sterbenden Patient_innen trotz Vorausplanung der Situation zu einem Notfallszenario werden, wenn die benötigten Medikamente nicht verfügbar sind und der/die zuständige Hausarzt/-ärztin nicht kurzfristig eingebunden werden kann:

Tabelle 2. Kategoriensystem

\begin{tabular}{lll}
\hline Hauptkategorien & Subkategorien & $\begin{array}{l}\text { Art der } \\
\text { Kategorienbildung }\end{array}$ \\
\hline 1 Notfallszenario & $\begin{array}{l}\text { Personenbezogene } \\
\text { Anlässe } \\
\text { Kontextbedingungen }\end{array}$ & Deduktiv \\
\hline 1.1 Personenbezogene & Krankheitsbilder & Deduktiv \\
Anlässe & Symptome & Deduktiv \\
& Ereignisse & Induktiv \\
\hline 1.2 Kontextbedingungen & Politisch-rechtlich & Deduktiv \\
& Ethisch & Deduktiv \\
& Organisatorisch- & Deduktiv \\
& strukturell & \\
& a) Intern & Induktiv \\
& b) extern & Induktiv \\
\hline
\end{tabular}




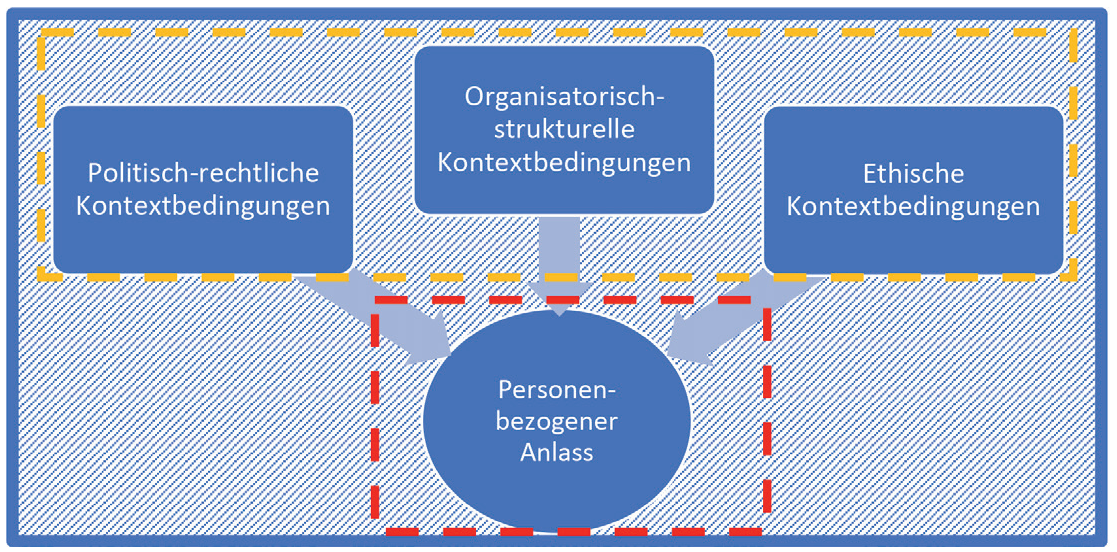

\section{Z2}

Abbildung 1. Konzeption von Notfallszenarien nach eigener Darstellung.
„[...] Man hat einen Palliativpatienten und der ist präfinal und sieht er wird unruhig und man sagt beim Hausarzt zu einer Zeit, wo er eben noch erreichbar ist: ,Bitte [...] geben Sie uns doch was an die Hand, wenn die Unruhe zunimmt, dass wir was haben. '[...] Und man bekommt nichts. [...] Man kann es noch so dringlich machen. Es wird gesagt: Ja, aber ich kann [...] nicht rauskommen und warten Sie erstmal ab.' Und dann ist die Situation da, die Bewohnerin ist dann in der Finalphase und ist derart unruhig und man hat nichts an der Hand, um [...] helfen zu können und dann zu einer Zeit, der Hausarzt ist nicht mehr zu erreichen. Und dann kann man das mit ansehen, [...] und die Frau so versterben lassen oder man kann es nicht mit ansehen [...]. Da gibt es dann bestimmt Situationen, wo Fachkräfte, die das dann nicht aushalten können, auch mal den Notdienst rufen" (Pflegefachperson 4).

Trotz der erheblichen Schwere der Situation stellt sich die auftretende Krise für die Pflegefachperson nicht zwangsläufig als Notfall dar. Der Krankheitsverlauf war erwartbar und wurde vorausgeplant, die Schmerzen hätten bei vorhandener Bedarfsmedikation durch Pflegefachpersonen gelindert werden können. Durch das Zusammenwirken von personenbezogenen Anlässen (starke Schmerzen) und unzureichenden Kontextbedingungen (fehlende Bedarfsmedikation, Nicht-Erreichbarkeit des/der Hausarztes/-ärztin) entsteht schließlich ein Notfallszenario, welches die Alarmierung des Rettungsdienstes zur Folge haben kann.

Als personenbezogene Anlässe wurden diverse pflegerisch- und medizinisch-relevante Veränderungen von Bewohner_innen zusammengefasst. Dazu gehören verschiedene Krankheitsbilder (z.B. Herzinfarkt, Schlaganfall), Symptome (z.B. Schmerzen, Luftnot, Schwäche, Übelkeit, Verwirrtheit, Vigilanzminderung, Krampfanfälle) und Ereignisse (z. B. Sturz, Suizidversuch). Von den insgesamt 73 gesammelten Merkmalen beziehen sich 35 auf personenbezogene Anlässe (siehe elektronisches Supplement ESM1).

Alle weiteren gesammelten 38 Merkmale lassen sich den Kontextbedingungen zuordnen, die ihrerseits weiter untergliedert werden können in a) politisch-rechtliche, b) organisatorisch-strukturelle, und c) ethische Kontextbedingungen. Notfallszenarien in Pflegeheimen können demnach als ein Zusammenwirken von personenbezogenem Anlass und Kontextbedingungen konzipiert werden.

\section{Identifizierung und Priorisierung von personenbezogenen Anlässen}

Die Teilnehmenden sammelten vor dem Hintergrund der eigenen Erfahrung die häufigsten personenbezogenen Anlässe in Pflegeheimen. Anschließend erfolgte eine Priorisierung hinsichtlich der Frage, für welche personenbezogenen Anlässe der größte Bedarf an Handlungsempfehlungen besteht (siehe Tab. 3).

Aus der Perspektive der Teilnehmenden besteht der größte Bedarf an Handlungsempfehlungen beim Umgang mit Stürzen, gefolgt von entgleisten Vitalwerten, auffälligem Verhalten und neurologischen Symptomen. Leblosigkeit, Luftnot, Schmerzen und akute Probleme von Palliativpatient_innen werden ebenfalls häufiger angeführt.

In der Praxis stellen sich die personenbezogenen Anlässe häufig nicht als klar abgrenzbare Phänomene dar. Bei den Teilnehmenden wird beispielsweise anhand der Diskussion zum Thema ,Sturz' deutlich, dass Ereignisse, Symptome und Krankheitsbilder zusammenfallen können:

„Manchmal weiß man ja auch nicht, ob eventuell eine Entgleisung der Vitalwerte dazu geführt hat, dass derjenige gestürzt ist. Der ist ja nicht immer gestürzt, weil er irgendwie gestolpert ist oder was auch immer, sondern aufgrund von einer Ischämie oder entgleisten Vitalwerten also zu Boden gegangen ist. Und da gibt es auch diese Situation, die man abklären muss" (Pflegefachperson 2).

Eine ähnliche Unschärfe findet sich bei den Themen , auffälliges Verhalten' und ,neurologische Symptome'. Dabei kann das auffällige Verhalten im Moment des Auftretens nicht immer unmittelbar auf eine Ursache zurückgeführt werden, insbesondere wenn die medizinische Vorgeschichte der Bewohnerin/des Bewohners nicht näher bekannt ist. 
Tabelle 3. Priorisierung von personenbezogenen Anlässen

\begin{tabular}{lc}
\hline Ereignis & Gesamt \\
\hline Sturz & 12 \\
Entgleiste Vitalwerte & 7 \\
Auffälliges Verhalten & 6 \\
Neurologische Symptome & 5 \\
Leblosigkeit & 4 \\
Luftnot & 3 \\
Schmerzen & 3 \\
Akute Probleme bei Palliativpatient_innen & 2 \\
Verschlechterung des Allgemeinzustand & 1 \\
Schockzustände & 1 \\
Husten & 1 \\
\hline Gesamt & 45 \\
\hline
\end{tabular}

„Verwirrtheit und Aggressivität, das könnte man natürlich unter neurologische Symptome oder Vigilanzminderung [einordnen]. Aber jetzt als doch extra Symptom nochmal. Und damit einhergehend vielleicht so ein permanentes Schreien [...], dass irgendwie Patienten da einfach ja unangenehm auffallen, aber sich keiner näher mit beschäftigen kann, will oder möchte. Warum, wieso, weshalb. Oder auch nicht zu einem Ergebnis kommt, weil es einfach nur ein Schreien gibt“" (Notfallsanitäter_in).

Aus der Perspektive der behandelnden Akteure existiert der personenbezogene Anlass nicht als gesicherte Diagnose und Krankheitsbild, sondern als Auffälligkeit, d.h.

Tabelle 4. Priorisierung von Kontextbedingungen

\begin{tabular}{|c|c|c|}
\hline \multicolumn{2}{|c|}{ Kontextbedingungen Aspekt } & \multirow{2}{*}{$\frac{\text { Gesamt }}{4}$} \\
\hline Politisch-rechtlich & $\begin{array}{l}\text { Eingeschränkte Handlungs- } \\
\text { spielräume Pflege }\end{array}$ & \\
\hline & Haftung, rechtliche Unsicherheit & 3 \\
\hline \multirow[t]{4}{*}{ Ethisch } & $\begin{array}{l}\text { Fehlende, uneindeutige Patienten- } \\
\text { verfügung }\end{array}$ & 5 \\
\hline & $\begin{array}{l}\text { Bevollmächtige Angehörige mit } \\
\text { unterschiedlichen Meinungen }\end{array}$ & 5 \\
\hline & Aktivistische Angehörige & 1 \\
\hline & $\begin{array}{l}\text { Fehlende Entscheidungshilfe } \\
\text { für Notfall }\end{array}$ & 1 \\
\hline \multirow[t]{2}{*}{$\begin{array}{l}\text { Organisatorisch- } \\
\text { strukturell - intern }\end{array}$} & $\begin{array}{l}\text { Ängste/Unsicherheiten } \\
\text { des Personals }\end{array}$ & 8 \\
\hline & Arbeitsverdichtung/Personalmangel & 5 \\
\hline \multirow[t]{3}{*}{$\begin{array}{l}\text { Organisatorisch- } \\
\text { strukturell - extern }\end{array}$} & $\begin{array}{l}\text { Kommunikation zwischen } \\
\text { den Akteuren }\end{array}$ & 8 \\
\hline & $\begin{array}{l}\text { Unterschiedliche Vorgaben Pflege- } \\
\text { Rettungsdienst }\end{array}$ & 1 \\
\hline & Ärztlicher Bereitschaftsdienst & 3 \\
\hline Gesamt & & $44^{*}$ \\
\hline
\end{tabular}

Anmerkung: *Bei der Priorisierung der Begleitumstände hat eine Person einen Klebepunkt nicht genutzt. Daher ist hier die Gesamtzahl im Vergleich zu den personenbezogenen Anlässe um $n=1$ geringer.
Abweichungen von einer angenommenen Normalität. In einer akuten Situation sind diese personenbezogenen Anlässe nicht immer eindeutig bestimmbar.

\section{Identifizierung und Priorisierung von Kontextbedingungen}

Kontextbedingungen sind vielschichtig und lassen sich in drei große Kategorien clustern: Ethisch, politisch-rechtlich und organisatorisch-strukturell. Nach der intensiven Sammlung zugehöriger Merkmale, erfolgte eine Priorisierung hinsichtlich der Frage, welche Kontextbedingungen am häufigsten für das Zustandekommen von Notfallszenarien verantwortlich sind (siehe Tab. 4).

Insgesamt wird den internen und externen organisatorisch-strukturellen Kontextbedingungen die größte Bedeutung für das Zustandekommen von Notfallszenarien zugeschrieben ( $n=24)$. Interne Bedingungen $(n=13)$ spielen dabei die größte Rolle, gefolgt von externen Bedingungen $(\mathrm{n}=11)$ sowie Kontextbedingungen aus dem ethischen $(n=11)$ und politisch-rechtlichen Bereich $(n=7)$.

\section{Organisatorisch-strukturelle Kontextbedingungen}

Für die Teilnehmenden stellen interne Bedingungen wie Ängste und Unsicherheiten des Personals und die Arbeitsverdichtung in der Pflege (v.a. durch Personalmangel) besonders relevante Faktoren dar, die auf Notfallszenarien Einfluss nehmen.

Die mangelhafte Kommunikation und Informationsweiterleitung zwischen allen behandelnden Akteuren (Pflege - Hausärzt_in - Rettungsdienst) wurde als besonders relevante externe Kontextbedingung identifiziert. Hinzu kommt die strukturell bedingte, eingeschränkte Erreichbarkeit des Ärztlichen Bereitschaftsdienstes sowie der Hausarztpraxen.

„Das ist das beste Szenario, wenn es dann in der Woche passiert. Passiert mir das am Wochenende oder Mittwochnachmittag, Freitagnachmittag, versuche ich die 116117 in der Regel, in so einem Fall nicht anzurufen, weil da muss ich sowieso bis zu drei Stunden warten, ehe ich einen Arzt überhaupt habe, und versuche erstmal eine Stunde zu telefonieren, bis ich überhaupt jemanden ans Telefon bekomme. Da gehe ich dann wirklich in dem Fall über den Rettungsdienst" (Pflegefachperson 1).

\section{Ethische Kontextbedingungen}

Im ethischen Bereich werden Unkenntnis und Uneindeutigkeit des Patientenwillens als wichtige Kennzeichen von Notfallszenarien genannt. Außerdem werden Situationen mit Angehörigen (mit oder ohne Vorsorgevollmacht) 
beschrieben, die Druck auf das Personal ausüben oder gegensätzliche Vorstellungen haben.

„Ja, ich glaube das gehört mehr zu den aktivistischen Angehörigen. Keine Einigkeit unter den Akteuren. Geht so ein bisschen darum, wenn man mehrere Angehörige hat und nur eine Bevollmächtigte und die sich mit den Geschwistern aber nicht verträgt" (Pflegefachperson 2).

\section{Politisch-rechtliche Kontextbedingungen}

Als besonders einschneidend erlebten die Teilnehmenden die eingeschränkten Handlungsspielräume und Entscheidungskompetenzen der Pflegefachpersonen (u.a. im Bereich der Bedarfs- und Notfallmedikation). Dies trägt zur Rechtsunsicherheit und Unzufriedenheit bei.

„Dann letztlich [...] mit der ganzen Entscheidungskompetenz und den Handlungsbefugnissen im Bereich der Pflege [...]. Nicht nur gibt es die ganzen Sachen (z.B. Medikamente, Salben) nicht, sondern selbst wenn das da wäre, dürfte man nicht oder das Beispiel, das Schmerzmedikament ist bei Bedarf für Schmerzen in der linken Schulter, der hat aber erst Schmerzen in der rechten Schulter, also geht das nicht. [...] Da ist eigentlich eine Handlungskompetenz da kraft Berufsausbildung und Erfahrung, aber das wird irgendwo extern eingeschränkt und man kommt gar nicht dahin und dann führt das zu so einer vermeintlichen Notfallsituation" (Ärzt_in 3).

Haftungsrechtliche Fragen auf Seiten der Pflegefachpersonen kennzeichnen ebenfalls häufig Notfallszenarien.

\section{Diskussion}

In dieser multimethodalen qualitativen Studie wurden Notfälle in Pflegeheimen analysiert. Aus interprofessioneller Perspektive stellen sich Notfallszenarien in Pflegeheimen als ein komplexes Zusammenspiel zwischen personenbezogenen Anlässen (pflegerisch- und medizinisch relevante Veränderungen) und organisatorisch-strukturellen, ethischen und politisch-rechtlichen Kontextbedingungen dar.

In der bisherigen Forschung werden Notfallsituationen in Pflegeheimen überwiegend als medizinische Krankheitsbilder wahrgenommen, die zu Krankenhauszuweisungen führen. Die berufsübergreifende und umfassende Betrachtung der Situationen fehlte bisher. Die Weiterentwicklung dieses Konstruktes als Notfallszenario erlaubt ein differenziertes Modell für das Zustandekommen und die systematische Bearbeitung von Notfallszenarien. Dort können Steuerungsinstrumente wie z.B. Handlungsempfehlungen ansetzen.
In unserer Studie konnten relevante personenbezogene Anlässe und Kontextbedingungen identifiziert und priorisiert werden. Die Ergebnisse unserer Studie bestätigen Befunde aus verschiedenen Reviews (Lemoyne et al., 2019), wonach traumatische Ereignisse (wie z. B. Stürze), Luftnot, ein veränderter mentaler Zustand sowie Erkrankungen des zentralen Nervensystems besonders bedeutsam sind. Interessant ist, dass die Teilnehmenden unserer Studie die personenbezogenen Anlässe eher auf der Ebene der Symptome und sichtbaren Wahrnehmungen beschreiben, während die quantitativen retrospektiven Daten aus der Forschungsliteratur bisweilen sehr konkrete Diagnosen als Ursachen für Notfälle ausweisen (Axon et al., 2015; Morphet et al., 2015). Folglich finden sich in unserer Studie beispielsweise nicht näher bestimmbares auffälliges Verhalten, Schmerzen oder eine Verschlechterung des Allgemeinzustandes. Diese Befunde lassen sich dadurch erklären, dass die Versorgungsakteure vor Ort häufig auf Grundlage der offensichtlichen Symptome eine Situationseinschätzung vornehmen müssen, ohne dass sie bereits eine gesicherte Diagnose feststellen können bzw. dürfen. Für die Entwicklung von Handlungsempfehlungen zur konkreten, praktischen Verbesserung des Notfallmanagements in Pflegeheimen ist dieser Befund äußerst relevant, muss es doch darum gehen, mit Handlungsempfehlungen an der Wahrnehmung der Akteure in der Notfallversorgung (Pflegefachpersonen, Ärzt_innen, Rettungsdienst) anzuschließen.

Die identifizierten Kontextbedingungen wirken stets auf die personenbezogenen Anlässe und nehmen deshalb im Notfallszenario eine essenzielle Rolle ein. Einzelne Aspekte sind im internationalen Kontext weitgehend bekannt, eine Kategorisierung und umfängliche Darstellung im deutschen Kontext fehlte bislang. So spielen beispielsweise die Erreichbarkeit von Hausärzt_innen, die Kommunikation zwischen den Versorgungsakteuren, Ängste und rechtliche Unsicherheiten der Pflegefachpersonen sowie unbekannte Behandlungswünsche von Patient_innen auch in anderen Studien eine wichtige Rolle (Bleckwenn et al., 2019; Lemoyne et al., 2019). Eine Besonderheit in Deutschland ist beispielsweise der Ärztliche Bereitschaftsdienst (ÄBD), welcher außerhalb der Öffnungszeiten von Hausarztpraxen eine eingeschränkte allgemeinmedizinische Versorgung sicherstellen soll. Unsere Ergebnisse deuten darauf hin, dass primär durch Wartezeiten bei der Kontaktaufnahme mit dem ÄBD über seine bundeseinheitliche Telefonnummer 116117 Rettungsdiensteinsätze hervorgerufen werden. Insgesamt könnte diesbezüglich eine Steigerung von Verbindlichkeit der hausärztlichen Versorgung in Pflegeheimen (z.B. konstante Erreichbarkeit von hausärztlichen Praxen, reguläre Heimvisiten) Abhilfe schaffen, um vermeidbare oder unerwünschte Krankenhauszuweisungen von Bewohner_innen zu reduzieren (Marshall, Clarke, Peddle \& Jensen, 2015; Pohontsch, Scherer \& Eisele, 2017). Zudem könnte die Implementierung von Heimarzt-Modellen (am Beispiel des Berliner Projekts) eine weitere Maßnahme darstellen. Beispielsweise konnten Kliniksaufenthalte von Bewohner_innen 
reduziert, die Menge von verordneten Medikamenten gesenkt und die Zufriedenheit der Betroffenen erhöht werden (Arbeitskreis Klinische Geriatrie der Ärztekammer Berlin, 2010).

Aus Sicht der Teilnehmenden führen die eingeschränkten rechtlichen Befugnisse der Pflegefachpersonen bei der Umsetzung ärztlicher Leistungen (z.B. Verbot der Gabe von nicht-verschreibungspflichtigen Medikamenten) zu Notfallszenarien. Hier geht es um die Frage, inwiefern die Delegation von ärztlichen Leistungen auf Pflegefachpersonen das Notfallmanagement verbessern kann. In Verbindung mit der ebenfalls benannten Problematik der teils unzureichenden Qualifikation des Personals in Pflegeheimen könnte eine Orientierung an internationalen Strukturen hilfreich sein. So werden dort speziell ausgebildete Fachkräfte, sogenannte Nurse Practitioner, mit spezialisiertem geriatrischen Wissen und koordinativen Aufgaben in Pflegeheimen eingesetzt. Studien zeigen, dass diese eine effektive Ressource darstellen, um Krankenhauseinweisungen von Bewohner_innen zu reduzieren (Christian \& Baker, 2009) und die Lebensqualität von Bewohner_innen zu verbessern (Arendts et al., 2018).

Im Einklang mit einer jüngst erschienen Studie von Blackwenn et al. (2019) legen unsere Ergebnisse nahe, dass Notfallszenarien in deutschen Pflegeheimen in hohem Maße durch solche unzureichenden Kontextbedingungen geprägt sind und häufig durch eine Verbesserung organisatorischer, rechtlicher und ethischer Rahmenbedingungen abgewendet werden könnten.

\section{Limitationen}

Die Datenerhebung fand in einem städtischen Versorgungsbereich Norddeutschlands statt und spiegelt folglich nur die Erfahrungen der dort handelnden Personen wider. Bei einer Verallgemeinerung der Ergebnisse müssen die lokalen Bedingungen berücksichtigt werden (z. B. was die Leistungsfähigkeit des ÄBD oder die hausärztliche Versorgung betrifft). Darüber hinaus gibt es folgende Einschränkungen bei der Auswahl der Teilnehmenden zu beachten: Einige der Teilnehmenden kannten sich schon aus dem bestehenden Arbeitskreis in Braunschweig, sodass dies die Redebeteiligung beeinflusst haben könnte. Aufgrund der sehr begrenzten zeitlichen Ressourcen von den Pflegefachpersonen, Ärzt_innen und Rettungsdienst wurde als Anreiz für die Termine eine Aufwandsentschädigung von $150 €$ gezahlt. Dies sollte darüber hinaus insbesondere die Beteiligung der Pflegefachpersonen sicherstellen, welche auch erreicht wurde. Die Kontaktaufnahme zu den Einrichtungen erfolgte primär über die Pflegedienstleitungen, weshalb diese überwiegend vertreten waren. Wünschenswert wäre ein ausgewogeneres Verhältnis zwischen Pflegefachpersonen mit und ohne Leitungsfunktion gewesen. Die Perspektive des Rettungsdienstes wurde nur durch eine / $\mathrm{n}$ Notfallsanitäter_in vertreten, welches im Vergleich $\mathrm{zu}$ den anderen Berufsgruppen eine deutliche Unterrepräsentation darstellt. Wissenschaftler_innen aus dem
NOVELLE Konsortium waren vor allem aufgrund der Verknüpfung zu den Teilprojekten beteiligt und nahmen in den Diskussionen eine eher passive Rolle ein.

\section{Schlussfolgerungen}

Notfälle in Pflegeheimen sind komplexe Szenarien, die durch verschiedene Kontextbedingungen verursacht und beeinflusst werden können. Die Einordnung als Notfallszenario kann einen strukturierten Umgang mit derartigen Situationen unterstützen. Die Ergebnisse liefern Hinweise, dass insbesondere Pflegefachpersonen auf Notfallszenarien in Pflegeheimen aufgrund der Kontextbedingungen unzureichend vorbereitet sind. Sie kommunizieren Unsicherheiten bezüglich des richtigen Umgangs mit Notfallsituationen, verfügen über ungenügende Ressourcen und fürchten juristische Konsequenzen. Die partizipative berufsübergreifende Entwicklung von Handlungsempfehlungen für Notfallszenarien kann ein Instrument sein, um Pflegefachpersonen und Ärzt_innen vor Ort mehr Handlungssicherheit zu geben. Hierfür sollten die Handlungsempfehlungen an dieser Notfallwahrnehmung anschließen und die rechtlichen, ethischen und organisatorischen Kontextbedingungen vor Ort berücksichtigen. Dies wird in weiteren Teilprojekten von NOVELLE adressiert und weiter bearbeitet.

\section{Elektronische Supplemente (ESM)}

Die elektronischen Supplemente sind mit der Online-Version dieses Artikels verfügbar unter https://oi.org/ 10.1024/1012-5302/a000804.

ESM1. Tabelle E1. Kennzeichen von Notfallszenarien in Pflegeeinrichtungen.

\section{Literatur}

Arbeitskreis Klinische Geriatrie der Ärztekammer Berlin. (2010). Geriatriekonzept Berlin 2010. Demografischer Wandel und medizinische Versorgung. Berliner Ärzte, (3), 15-20.

Arendts, G., Deans, P., O'Brien, K., Etherton-Beer, C., Howard, K., Lewin, G. et al. (2018). A clinical trial of nurse practitioner care in residential aged care facilities. Archives of gerontology and geriatrics, 77, 129-132.

Axon, R. N., Gebregziabher, M., Craig, J., Zhang, J., Mauldin, P. \& Moran, W. P. (2015). Frequency and costs of hospital transfers for ambulatory care-sensitive conditions. The American Journal of Managed Care, 21(1), 51 - 59.

Behringer, W., Buergi, U., Christ, M., Dodt, C. \& Hogan, B. (2013) Fünf Thesen zur Weiterentwicklung der Notfallmedizin in Deutschland, Österreich und der Schweiz. Notfall + Rettungsmedizin, 16 (8), $625-626$.

Bleckwenn, M., Bell, L., Schnakenberg, R., Weckbecker, K. \& Klaschik, M. (2019). Ambulante Notfallversorgung von Pflegeheimbewohner: Ein Status Quo aus pflegerischer Sicht. [Outpatient Emergency Care for Nursing Home Residents: A Status Quo From a Nursing Perspective]. Gesundheitswesen (Bundesverband der Ärzte des Öffentlichen Gesundheitsdienstes (Germany)), $81(06), 486-491$. 
Bohnsack, R. (2000). Gruppendiskussion. In U. Flick, E. v. Kardorff \& I. Steinke (Hrsg.), Qualitative Forschung. Ein Handbuch. (S.369 - 384). Reinbek: Rowohlt Taschenbuch Verlag.

Burke, R. E., Rooks, S. P., Levy, C., Schwartz, R. \& Ginde, A. A. (2015). Identifying Potentially Preventable Emergency Department Visits by Nursing Home Residents in the United States. Journal of American Medical Directors Association, 16(5), 395 - 399.

Chess, D., Whitman, J. J., Croll, D. \& Stefanacci, R. (2018). Impact of after-hours telemedicine on hospitalizations in a skilled nursing facility. The American journal of managed care, 24(8), 385 - 388.

Christian, R. \& Baker, K. (2009). Effectiveness of Nurse Practitioners in nursing homes: a systematic review. JBI Library of Systematic Reviews, 7(30), $1333-1352$.

Dwyer, R., Gabbe, B., Stoelwinder, J. \& Lowthian, J. (2014). A systematic review of outcomes following emergency transfer to hospital for residents of aged care facilities. Age and ageing, 43(6), $759-766$.

Dyntar, D., Geschwindner, H., Theill, N., Negatsch, S., Meier, A., Gmünder, R. et al. (2018). Gute ambulante Versorgung verzögert den Heimeintritt bei Menschen mit Demenz in der Stadt Zürich. Praxis, 107(9-10), $505-511$.

Fernandez, H. M., Callahan, K. E., Likourezos, A. \& Leipzig, R. M. (2008). House staff member awareness of older inpatients' risks for hazards of hospitalization. Archives of internal medicine, 168(4), $390-396$.

Flick, U., Kardorff, E.v. \& Steinke, I. (2012). Was ist qualitative Forschung? Einleitung und Überblick. In U. Flick, E.v. Kardorff \& I. Steinke (Hrsg), Qualitative Forschung. Ein Handbuch. (9. Auflage, S.13 - 29). Hamburg: Rowohlt Taschenbuch Verlag.

Graverholt, B., Riise, T., Jamtvedt, G., Ranhoff, A. H., Krüger, K. \& Nortvedt, M. W. (2011). Acute hospital admissions among nursing home residents: a population-based observational study. BMC Health Services Research, 11(1), 126.

Kada, O., Janig, H., Likar, R. \& Pinter, G. (2013). Versorgung optimieren, vermeidbare Krankenhaustransporte reduzieren. In G. Pinter, R. Likar, W. Schippinger, H. Janig, O. Kada \& K. Cernic (Eds.), Geriatrische Notfallversorgung: Strategien und Konzepte (S. 227 -252). Wien: Springer.

Kuckartz, U. (2016). Qualitative Inhaltsanalyse. Methoden, Praxis, Computerunterstützung (3., überarbeitete Auflage). Weinheim und Basel: Beltz Juventa.

Lemoyne, S. E., Herbots, H. H., Blick, D. D., Remmen, R., Monsieurs, K. G. \& van Bogaert, P. (2019). Appropriateness of transferring nursing home residents to emergency departments: a systematic review. BMC Geriatrics, 19(1), 17.

Marshall, E., Clarke, B., Peddle, S. \& Jensen, J. (2015). Care by Design: New model of coordinated on-site primary and acute care in long-term care facilities. Canadian Family Physician, 61, $129-134$.

Mayring, P. (2008). Qualitative Inhaltsanalyse. Grundlagen und Techniken (10., neu ausgestattete Aufl.). Weinheim und Basel: Beltz.

Morphet, J., Innes, K., Griffiths, D. L., Crawford, K. \& Williams, A. (2015). Resident transfers from aged care facilities to emergency departments: can they be avoided? Emergency Medicine Australasia, 27(5), 412-418.

Ouslander, J. (2019). Strategies to reduce potentially avoidable hospitalisations among long-term care facility residents. BMJ Quality \& Safety, 28(7), 515-519.

Ouslander, J. G., Lamb, G., Perloe, M., Givens, J. H., Kluge, L., Rutland, T. et al. (2010). Potentially avoidable hospitalizations of nursing home residents: frequency, causes, and costs: [see editorial comments by Drs. Jean F. Wyman and William R. Hazzard, pp 760 - 761]. Journal of the American Geriatrics Society, 58(4), $627-635$.

Pohontsch, N. J., Scherer, M. \& Eisele, M. (2017). (In-)formal caregivers' and general practitioners' views on hospitalizations of people with dementia - an exploratory qualitative interview study. BMC Health Services Research, 17(1), 530
Przyborski, A. \& Riegler, J. (2010). Gruppendiskussion und Fokusgruppe. In G. Mey \& K. Mruck (Hrsg), Handbuch Qualitative Forschung in der Psychologie (S.436-448). Wiesbaden: VS Verlag für Sozialwissenschaften.

Robert Koch-Institut. (2015). Gesundheit in Deutschland. Gesundheitsberichterstattung des Bundes. Gemeinsam getragen von RKI und Destatis. Verfügbar unter https://www.gbe-bund.de/ pdf/GESBER2015.pdf [07.12.2020].

Ruddat, M. (2012). Auswertung von Fokusgruppen mittels Zusammenfassung zentraler Diskussionsaspekte. In M. Schulz, B. Mack \& O. Renn (Hrsg.), Fokusgruppen in der empirischen Sozialwissenschaft (S.195 - 206). Wiesbaden: VS Verlag für Sozialwissenschaften.

Seeger, I., Ramos, A. L. \& Hoffmann, F. (2017). Ambulante Notfallversorgung von Pflegeheimbewohnern. Zeitschrift für Gerontologie und Geriatrie, 51(6), 650 - 655.

Statista. (2020). Pflege in Deutschland - Zahlen und Statistiken. Verfügbar unter https://de.statista.com/themen/785/pflegein-deutschland/ [07.12.2020].

Tiedtke, J., Lang, F., Schulze, U., Hasseler, M., Howe, J., Haase, T. et al. (2014). Sterben in stationären Pflegeeinrichtungen. Gießen: Psychosozial-Verlag.

Wang, H. E., Shah, M. N., Allman, R. M. \& Kilgore, M. (2011). Emergency department visits by nursing home residents in the United States. Journal of American Geriatrics Society, 59(10), $1864-1872$

\section{Historie}

Manuskripteingang: 03.09.2020

Manuskript angenommen: 06.04.2021

Onlineveröffentlichung: 28.04.2021

\section{Förderung}

Die Studie wurde im Rahmen des Forschungsprojektes „Sektorenübergreifendes \& integriertes Notfall- und Verfügungsmanagement für die letzte Lebensphase (NOVELLE) vom Innovationsfonds des Gemeinsamen Bundesausschuss (G-BA) unter dem Förderkennzeichen 01NVF18007 gefördert.

\section{Danksagung}

Ein großer Dank geht an die Studienteilnehmer_innen für ihre Offenheit und Bereitschaft zur Mitarbeit sowie an Dorothea Bünsow, Bianca Steiner und Birgit Hartleb für die Unterstützung bei der Organisation und Dokumentation der Gruppendiskussionen.

\section{Autorenschaft}

Beitrag zur Konzeption oder zum Design der Arbeit:JP, CB, SF, AG, $\mathrm{MH}, \mathrm{NSch}, \mathrm{JB}, \mathrm{SSch}$

Beitrag bei der Erfassung, Analyse oder der Interpretation der Daten:JP, CB, SF, AG, MH, NSch, JB, SSch

Manuskripterstellung oder kritische Überarbeitung von wichtigen intellektuellen Inhalten des Manuskripts: JP, CB, SF, AG, MH,

NSch, JB, SSch

Genehmigung der letzten Version des Manuskripts zur Publikation: JP, CB, SF, AG, MH, NSch, JB, SSch

Bereitschaft, für alle Aspekte der Arbeit Verantwortung zu übernehmen: JP, CB, SF, AG, MH, NSch, JB, SSch

\section{ORCID}

Juliane Poeck

(D) https://orcid.org/0000-0002-7814-7972 


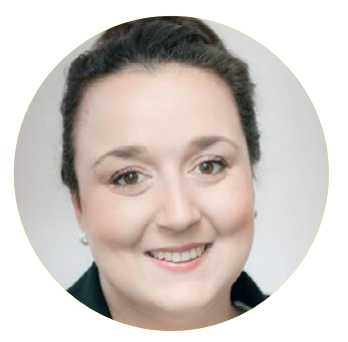

Juliane Poeck, M.Sc.

Institut für Allgemeinmedizin

Universitätsklinikum Jena

Bachstraße 18k

07743 Jena

Deutschland

juliane.poeck@med.uni-jena.de

Was war die größte Herausforderung bei Ihrer Studie?

Pflegefachpersonen für die Teilnahme zu gewinnen. Die Kommunikation verlief meist über die Heim- und Pflegedienstleitungen, die Ressourcen in den Einrichtungen waren sehr begrenzt.

Was wünschen Sie sich bezüglich der Thematik für die Zukunft?

Mehr aktive Forschungsprojekte mit Pflegefachpersonen für

Pflegefachpersonen.

Was empfehlen Sie zum Weiterlesen/Vertiefen?

Lemoyne, Herbots, Blick, Remmen, Monsieurs \& van Bogaert

(2019). Appropriateness of transferring nursing home residents to emergency departments: a systematic review. Siehe Literatur. 